J. Austral. Math. Soc. 25 (Series A) (1978), 29-34

\title{
ON GENERALIZED BOREL SETS
}

\author{
W. F. PFEFFER
}

(Received 10 January; revised 30 June 1977)

Communicated by J. Virsik

\begin{abstract}
A certain natural extension $\mathscr{B}$ of the Borel $\sigma$-algebra is studied in generalized weakly $\theta$-refinable spaces. It is shown that a set belongs to $\mathscr{B}$ whenever it belongs to $\mathscr{B}$ locally. From this it is derived that if $\boldsymbol{\aleph}_{\alpha}$ is an uncountable regular cardinal which is not two-valued measurable, then the space of all ordinals less than $\omega_{\alpha}$ is more complicated than a union of less than $\aleph_{\alpha}$ weakly $\theta$-refinable subspaces.
\end{abstract}

Subject classification (Amer. Math. Soc. (MOS) 1970): primary 28A05, $54 \mathrm{D} 20$, secondary $28 \mathrm{~A} 10$

Keywords: Borel sets, measurable cardinal, weakly $\theta$-refinable, regular ordinal

Given a set $A$, we shall denote by $|A|$ the cardinality of $A$ and by $\exp A$ the family of all subsets of $A$. Throughout, by $\aleph$ we shall denote an uncountable cardinal.

Defintion 1 . Let $Z$ be a set. A family $\mathscr{A} \subset \exp Z$ is called an $\aleph$-algebra in $Z$ if

(i) $Z \in \mathscr{A}$;

(ii) $A \in \mathscr{A} \Rightarrow Z-A \in \mathscr{A}$;

(iii) $\left(\left\{A_{\alpha}: \alpha \in T\right\} \subset \mathscr{A}\right.$ and $\left.|T|<\aleph\right) \Rightarrow \bigcup\left\{A_{\alpha}: \alpha \in T\right\} \in \mathscr{A}$.

Defintion 2. Let $\mathscr{A}$ be an $\aleph$-algebra in a set $Z$. A function $\mu: \mathscr{A} \rightarrow[0,+\infty]$ is called an $\aleph$-measure on $\mathscr{A}$ if $\mu(\varnothing)=0$ and

$$
\mu\left(\mathrm{U}\left\{A_{\alpha}: \alpha \in T\right\}\right)=\Sigma\left\{\mu\left(A_{\alpha}\right): \alpha \in T\right\}
$$

for each disjoint family $\left\{A_{\alpha}: \alpha \in T\right\} \subset \mathscr{A}$ with $|T|<\aleph$.

Thus in our terminology, a $\sigma$-additive measure on a $\sigma$-algebra will be called an $\aleph_{1}$-measure on an $\aleph_{1}$-algebra.

Let $\mathscr{A}$ be an $\aleph$-algebra in a set $Z$ and let $\mu$ be an $\aleph$-measure on $\mathscr{A}$. We shall say that $\mu$ is complete if $A \in \mathscr{A}$ whenever there is a $B \in \mathscr{A}$ such that $A \subset B$ and $\mu(B)=0$. We shall say that $\mu$ is saturated if $A \in \mathscr{A}$ whenever $A \cap B \in \mathscr{A}$ for each $B \in \mathscr{A}$ with $\mu(B)<+\infty$.

An uncountable cardinal $\$$ is called measurable if there is a set $Z$ with $|Z|=\$$ and an $\aleph$-measure $\mu$ on $\exp Z$ such that $\mu(Z)=1$ and $\mu(\{z\})=0$ for each $z \in Z$. 
If the measure $\mu$ takes only values 0 and 1 , the cardinal $\aleph$ is called two-valued measurable. The basic properties of measurable and two-valued measurable cardinals which do not involve axiomatic set theory are proved in Ulam (1930); more recent results can be found, for example, in Dickmann (1975, Chapter 0, Section 4).

Unless specified otherwise, throughout, $X$ will be an arbitrary topological space. By $\mathscr{G}$ we shall denote the family of all open subsets of $X$. Let $Y \subset X$. A collection $\left\{A_{\alpha}: \alpha \in T\right\} \subset \exp X$ is called separated in $Y$ if $\left\{A_{\alpha}: \alpha \in T\right\} \subset \exp Y$ and there is a family $\left\{G_{\alpha}: \alpha \in T\right\} \subset \mathscr{G}$ such that $\left\{G_{\alpha} \cap Y: \alpha \in T\right\}$ is a disjoint collection and $A_{\alpha} \subset G_{\alpha}$ for each $\alpha \in T$.

Definttion 3. An $\aleph$-algebra $\mathscr{A}$ in $X$ is called complete (abbreviated as $c \aleph-$ algebra) if $\bigcup\left\{A_{\alpha}: \alpha \in T\right\} \in \mathscr{A}$ for every collection $\left\{A_{\alpha}: \alpha \in T\right\} \subset \mathscr{A}$ which is separated in some $Y \in \mathscr{A}$.

Clearly, $\exp X$ is a c\$-algebra in $X$, and the intersection of any nonempty family of c\$-algebras in $X$ is again a c $N$-algebra in $X$. Thus we can define the Borel cא-algebra in $X$ as the smallest $\mathrm{c} \aleph-a l g e b r a \mathscr{B}_{\mathbb{N}}$ in $X$ containing $\mathscr{G}$. The elements of $\mathscr{B}_{\mathcal{N}}$ will be called cא-Borel subsets of $X$.

The next two propositions indicate that $\mathbf{c}$-Borel subsets occur quite naturally.

Proposition 1. Let $\mathscr{A}$ be an $\aleph$-algebra in $X$ containing $\mathscr{G}$ and let $\mu$ be a complete and saturated $\aleph$-measure on $\mathscr{A}$. If $X$ contains no discrete subspace of measurable cardinality, then $\mathscr{A}$ is complete and so $\mathscr{B}_{\aleph} \subset \mathscr{A}$.

PRoOF. Let $\left\{A_{\alpha} \neq \varnothing: \alpha \in T\right\} \subset \mathscr{A}$ be separated in some $Y \in \mathscr{A}$ and let $A=\bigcup\left\{A_{\alpha}: \alpha \in T\right\}$. Choose $B \in \mathscr{A}$ with $\mu(B)<+\infty$ and $\left\{G_{\alpha}: \alpha \in T\right\} \subset \mathscr{G}$ such that $\left\{G_{\alpha} \cap Y: \alpha \in T\right\}$ is a disjoint family and $A_{\alpha} \subset G_{\alpha} \cap Y$ for each $\alpha \in T$. Let $T_{0}=\left\{\alpha \in T: \mu\left(G_{\alpha} \cap Y \cap B\right)=0\right\}$ and $B_{0}=\bigcup\left\{G_{\alpha} \cap Y \cap B: \alpha \in T_{0}\right\}$. Suppose that $\mu\left(B_{0}\right)>0$. Because the sets $G_{\alpha} \cap Y \cap B$ are open in $Y \cap B$ and disjoint, we can define an $\aleph$-measure $\nu$ on $\exp T_{0}$ by letting

$$
\nu\left(T^{\prime}\right)=\frac{1}{\mu\left(B_{0}\right)} \mu\left(\bigcup\left\{G_{\alpha} \cap Y \cap B: \alpha \in T^{\prime}\right\}\right)
$$

for each $T^{\prime} \subset T_{0}$. Since $\aleph>\aleph_{0}$, it follows from Dickman (1975, Lemma 0.4.12, p. 36) that $T_{0}$ contains a set $T_{1}$ of measurable cardinality. Choosing $x_{\alpha} \in A_{\alpha}$ for each $\alpha \in T_{1}$, we obtain a discrete subspace $X_{1}=\left\{x_{\alpha}: \alpha \in T_{1}\right\}$ of $X$ with $\left|X_{1}\right|=\left|T_{1}\right|$. This contradiction shows that $\mu\left(B_{0}\right)=0$. By the completeness of $\mu$,

$$
\bigcup\left\{A_{\alpha} \cap B: \alpha \in T_{0}\right\} \in \mathscr{A} \text {. }
$$

Because $\mu(B)<+\infty$, we have $\left|T-T_{0}\right| \leqslant \aleph_{0}<\aleph$. Hence

$$
A \cap B=\left(\bigcup\left\{A_{\alpha} \cap B: \alpha \in T_{0}\right\}\right) \cup\left(\bigcup\left\{A_{\alpha} \cap B: \alpha \in T-T_{0}\right\}\right)
$$

belongs to $\mathscr{A}$. Since $\mu$ is saturated, $A \in \mathscr{A}$. 
REMARK 1. From the previous proof it is clear that if $\mu$ is a two-valued measure, we can replace "measurable" by "two-valued measurable" in Proposition 1: we only need to apply Dickmann (1975, Theorem $0.4 .25(4)$, p. 39).

A set $A \subset X$ is called $\aleph$-Lindelöf if every open cover of $A$ contains a subcover whose cardinality is less than $\aleph$. Thus an ordinary Lindelöf set is $\aleph_{1}$-Lindelöf. We shall denote by $\mathscr{F}_{k}$ the family of all closed $\aleph$-Lindelöf subsets of $X$.

Let $\mathscr{A}$ be an $\aleph$-algebra in $X$ containing $\mathscr{G}$. An $\aleph$-measure $\mu$ on $\mathscr{A}$ is called inner regular if

$$
\mu(A)=\sup \left\{\mu(C): C \in \mathscr{F}_{N}, C \subset A\right\}
$$

for each $A \in \mathscr{A}$ with $\mu(A)<+\infty$.

Proposition 2. Let $\mathscr{A}$ be an $\aleph$-algebra in $X$ containing $\mathscr{G}$ and let $\mu$ be a complete and saturated $\aleph$-measure on $\mathscr{A}$. If $\mu$ is inner regular, then $\mathscr{A}$ is complete and so $\mathscr{B}_{N} \subset \mathscr{A}$.

Proof. Using the same notation as in the proof of Proposition 1, it clearly suffices to show that $\mu\left(B_{0}\right)=0$. If $C \in \mathscr{F}_{\mathbb{N}}$ and $C \subset B_{0}$, then

$$
C \subset \bigcup\left\{G_{\alpha} \cap Y \cap B: \alpha \in S\right\}
$$

where $S \subset T_{0}$ with $|S|<\aleph$. Hence $\mu(C)=0$ for each $C \in \mathscr{F}_{\aleph}$ for which $C \subset B_{0}$. By the inner regularity of $\mu, \mu\left(B_{0}\right)=0$.

The Borel $\aleph$-algebra in $X$ is defined as the smallest $\aleph$-algebra in $X$ containing $\mathscr{G}$. Thus the Borel $\aleph$-algebra in $X$ is contained in $\mathscr{B}_{\aleph}$ but, in general, it is not complete. If $X$ is a free union of subspaces $X_{\alpha}$, then it is easy to see that the Borel ck-algebra in $X$ is isomorphic to the direct product of the Borel c\$-algebras in $X_{\alpha}^{\prime}$ 's. This is not correct if the Borel c $\aleph$-algebras are replaced by the Borel $\aleph$-algebras. The situation is well illustrated by the following example.

EXAMPLE 1. Let $T$ be the discrete space of all countable ordinals and let $X=T \times[0,1]$. According to Natanson (1957, Chapter 15, Section 2), for each $\alpha \in T$ there is a set $A_{\alpha} \subset[0,1]$ whose characteristic function belongs to the Baire class $\alpha$. Thus the set $A=\bigcup\left\{(\alpha) \times A_{\alpha}: \alpha \in T\right\}$ is not a Borel subset of $X$. Obviously, $A \in \mathscr{B}_{\mathrm{si}_{1}}$.

A set $A \subset X$ is called locally ç-Borel if for each $x \in X$ there is a neighborhood $U$ of $x$ such that $A \cap U \in \mathscr{B}_{\mathbb{N}}$. The family of all locally cN-Borel subsets of $X$ will be denoted by $\mathscr{L}_{\mathrm{N}}$. Obviously, $\mathscr{B}_{\mathrm{k}} \subset \mathscr{L}_{\mathrm{k}}$ and, in general, this inclusion is proper (see the Corollary to Proposition 3). If $\mathscr{B}_{\mathbb{N}}=\mathscr{L}_{\mathbb{N}}$, the space $X$ is called $\aleph$-saturated.

If $\mathscr{V} \subset \exp X$ and $x \in X$, let st $(x, \mathscr{V})=\{V \in \mathscr{V}: x \in V\}$.

Definition 4. The space $X$ is called $\aleph$-weakly $\theta$-refinable if each open cover of $X$ has an open refinement $\mathscr{V}=\bigcup\left\{\mathscr{V}_{\alpha}: \alpha \in T\right\}$ such that $|T|<\aleph$ and for each $x \in X$ there is an $\alpha_{x} \in T$ such that $\operatorname{st}\left(x, \mathscr{V}_{\alpha_{\alpha}}\right)$ is nonempty and finite. 
We note that $X$ is weakly $\theta$-refinable in the sense of Bennett and Lutzer (1972) if and only if it is $\aleph_{1}$-weakly $\theta$-refinable.

TheOREM. Let $X$ be $\aleph$-weakly $\theta$-refinable. Then $X$ is $\aleph$-saturated.

Proof. Let $A \in \mathscr{L}_{w}$. For each $x \in X$ choose an open neighborhood $U_{x}$ of $x$ so that $A \cap U_{x} \in \mathscr{B}_{B}$. Let $\mathscr{V}=\bigcup\left\{\mathscr{V}_{\alpha}: \alpha \in T\right\}$ be an open refinement of $\left\{U_{x}: x \in X\right\}$ such that $|T|<\boldsymbol{N}$ and given $x \in X$, there is an $\alpha_{x} \in T$ for which st $\left(x, \mathscr{V}_{\alpha_{z}}\right)$ is nonempty and finite. Because the sets $\left\{x \in X:\left|\operatorname{st}\left(x, \mathscr{V}_{\alpha}\right)\right| \geqslant k\right\}, \alpha \in T, k=1,2, \ldots$, are open, the sets

$$
X_{\alpha, k}=\left\{x \in X:\left|\operatorname{st}\left(x, \mathscr{V}_{\alpha}\right)\right|=k\right\}
$$

are $\mathbf{c}$-Borel. Clearly,

$$
\bigcup\left\{X_{\alpha, k}: \alpha \in T, k=1,2, \ldots\right\}=X .
$$

Let $\mathscr{W}_{\alpha, k}$ consist of all sets $A \cap X_{\alpha, k} \cap V_{1} \cap \ldots \cap V_{k}$ where $V_{1}, \ldots, V_{k}$ are distinct elements of $\mathscr{V}_{\alpha}$. Then $\mathscr{W}_{\alpha, k}$ is separated in $X_{\alpha, k}$ and $\bigcup\left\{W: W \in \mathscr{W}_{\alpha, k}\right\}=A \cap X_{\alpha, k}$. Since $\mathscr{W}_{\alpha, k} \subset \mathscr{B}_{\aleph}$, we have $A \cap X_{\alpha, k} \in \mathscr{B}_{\aleph}$ for $\alpha \in T$ and $k=1,2, \ldots$. The theorem follows.

Throughout, let $\kappa$ be an uncountable ordinal. By $W$ we shall denote the set of all ordinals less than $\kappa$ equipped with the order topology, and we let $\kappa=|W|$. The family of all closed cofinal subsets of $W$ is denoted by $\mathscr{H}$. Thus if $\kappa$ is a regular ordinal, then $\mathscr{H}$ consists of all closed sets $F \subset W$ for which $|F|=\aleph$.

LeMma Let $\kappa$ be a regular ordinal, $\left\{F_{\alpha}: \alpha \in T\right\} \subset \mathscr{H}$, and let $F=\bigcap\left\{F_{\alpha}: \alpha \in T\right\}$. If $|T|<\aleph$ then $F \in \mathscr{H}$.

Proof. Using the interlacing lemma (see Kelley, 1955, Chap. 4, Prob. E, (a)) in $W$, it is easy to see that the lemma is correct if $|T|=2$. By induction it is correct whenever $|T|<\boldsymbol{\aleph}_{0}$. Let $\boldsymbol{\aleph}_{0} \leqslant m<\boldsymbol{\aleph}$ and suppose that the lemma is correct if $|T|<m$. Let $\xi$ be the initial ordinal for $m$ and let $T=\{\alpha: \alpha<\xi\}$. Replacing $F_{\alpha}$ by $\cap\left\{F_{\beta}: \beta \leqslant \alpha\right\}$, we may assume that $F_{\alpha} \subset F_{\beta}$ for each $\beta<\alpha<\xi$. Given $\gamma<\kappa$, there are $\gamma_{\alpha} \in F_{\alpha}$ such that $\gamma<\gamma_{\alpha}<\gamma_{\beta}$ for each $\alpha<\beta<\xi$. Let $\delta=\sup \left\{\gamma_{\alpha}: \alpha<\xi\right\}$. Since $\kappa$ is a regular ordinal, $\delta<\kappa$. It follows that $\delta \in F$ and so $F \in \mathscr{H}$.

Let $\mathscr{A}$ consist of all sets $A \subset W$ such that either $A$ or $W-A$ contain a set $F \in \mathscr{H}$. For $A \in \mathscr{A}$ let $\mu(A)=1$ if $A$ contains a set $F \in \mathscr{H}$ and $\mu(A)=0$ otherwise. The next proposition follows immediately from the lemma.

Proposition 3. Let $\kappa$ be a regular ordinal. Then the family $\mathscr{A}$ is an $\aleph$-algebra in $W$ containing all open subsets of $W$ and $\mu$ is a complete $\aleph$-measure on $\mathscr{A}$.

COROLLARY. Let $\kappa$ be a regular ordinal such that the cardinal $\aleph$ is not two-valued measurable. Then $W$ is not $\$$-saturated and hence not $\$$-weakly $\theta$-refinable. 
Proof. The space $W$ is Hausdorff and each $x \in W$ has a neighborhood $U$ with $|U|<\aleph$. Thus $\mathscr{L}_{N}=\exp W$. Because the cardinal $\aleph$ is not two-valued measurable, $\mathscr{A} \neq \exp W$. Being finite, the $\aleph$-measure $\mu$ is saturated. By Proposition 1 and Remark $1, \mathscr{B}_{\mathbb{N}} \subset \mathscr{A}$. The corollary follows from the theorem.

Bennett and Lutzer (1972) proved that $W$ is weakly $\theta$-refinable if and only if it is paracompact (Theorem 11). A simple modification of this proof will show that $W$ is not $\aleph$-weakly $\theta$-refinable for any uncountable regular ordinal $\kappa$.

Remark 2. If the cardinal $\aleph$ is two-valued measurable, we cannot use Proposition 1 to show that $\mathscr{B}_{K} \subset \mathscr{A}$. However, K. Prikry kindly pointed out to the author that $\mathscr{A} \neq \exp W$ for any uncountable regular ordinal $\kappa$. Indeed, this is clear if $\kappa=\omega_{1}$, for $\aleph_{1}$ is not measurable (see Ulam, 1930, Theorem (A)). If $\kappa>\omega_{1}$ then each closed cofinal subset of $W$ contains an ordinal $\alpha$ cofinal with $\omega_{0}$ and also an ordinal $\beta$ cofinal with $\omega_{1}$. Hence if $B$ is the set of all ordinals $\alpha \in W$ cofinal with $\omega_{0}$, then $B \notin \mathscr{A}$.

We shall close this paper with an example indicating the necessity of the cardinality assumption in Proposition 1.

EXAMPLE 2. Let $\boldsymbol{\aleph}$ be a two-valued measurable cardinal and let $Z$ be a discrete space of cardinality $\aleph$. Denote by $\nu$ a two-valued $\aleph$-measure on $\exp Z$ such that $v(Z)=1$ and $\nu(\{z\})=0$ for each $z \in Z$. If $\kappa$ is the initial ordinal for $\kappa$, then $\kappa$ is regular (see Ulam (1930)). Thus we can define the $\aleph$-measure $\mu$ in $W$ as in Proposition 3. Let $X=W \times Z$. For $C \subset X$ and $\alpha \in W$ set $C^{\alpha}=\{z \in Z:(\alpha, z) \in C\}$ and $C^{\prime}=\left\{\alpha \in W: \nu\left(C^{\alpha}\right)=1\right\}$. Denote by $\mathscr{C}$ the family of those $C \subset X$ for which $C^{\prime} \in \mathscr{A}$ and let $\lambda(C)=\mu\left(C^{\prime}\right)$ for each $C \in \mathscr{C}$. It is easy to see that $\mathscr{C}$ is an $\aleph$-algebra in $X$ and that $\lambda$ is a complete two-valued $\aleph$-measure on $\mathscr{C}$. Let $G \subset X$ be open and let $\alpha \in G^{\prime}$ be a limit ordinal. For each $\beta<\alpha$ let

$$
A_{\beta}=\left\{z \in G^{\alpha}:(\beta, \alpha] \times\{z\} \subset G\right\} .
$$

Since $G$ is open, $G^{\alpha}=\bigcup\left\{A_{\beta}: \beta<\alpha\right\}$. It follows that $\nu\left(A_{\beta}\right)=1$ for some $\beta<\alpha$. Consequently, $(\beta, \alpha] \subset G^{\prime}$ and $G^{\prime}$ is open. Therefore, $\mathscr{G} \subset \mathscr{C}$. Choose $A \subset W$ for which $A \notin \mathscr{A}$ (see Remark 2). Clearly, we can consider $Z$ as $W$ with the discrete topology. Let $B=\{(\alpha, z) \in X: \alpha \in A$ and $z>\alpha\}$. Then $B^{\prime}=A$ and thus $B \notin \mathscr{C}$. However,

$$
B=\bigcup\{(A \cap[0, z)) \times\{z\}: z \in Z\}
$$

from which it follows that $B \in \mathscr{B}_{\mathrm{N}}$.

\section{References}

H. R. Bennett and D. J. Lutzer (1972), "A note on weak $\theta$-refinability", Gen. Topology Appl. 2, 49-54.

M. A. Dickmann (1975), Large Infinitary Languages (North-Holland, Amsterdam). 
J. L. Kelley (1955), General Topology (Van Nostrand, New York).

I. P. Natanson (1957), Theory of Functions of Real Variable (Ungar, New York, 1964).

S. Ulam (1930), "Zur Masstheorie in der allgemeinen Mengenlehre", Fund. Math. 16, 140-150.

Department of Mathematics

University of California

Davis, California 95616

USA 